\title{
COMMUNICATIONS
}

\section{DRAINAGE CHANNELS IN THE TRABECULAR WALL OF SCHLEMM'S CANAL*†}

\author{
BY
}

\section{J. S. SPEAKMAN}

Department of Ophthalmology, University of Toronto, and Department of Pathology, Institute of Ophthalmology, London

THE detailed structure of the trabecular wall of Schlemm's canal in man has previously been illustrated by means of teased flat preparations and thin paraffin sections (Speakman, 1959a, b). Features of interest were deep grooves lined with endothelial cells, openings 5-10 $\mu$ in diameter which communicated with deeper spaces in the meshwork, and numerous vacuoles or spaces in the endothelial cells lining the canal. Gross vacuolation was also found in the corneal endothelium, where it was shown to be the result of post-mortem degeneration, and it was suggested that the trabecular endothelium might undergo similar degenerative changes. In order to interpret more accurately the significance of the endothelial cell morphology, a further examination of this region has been made in both monkey and human eyes employing rapid fixation to prevent post-mortem artefacts.

\section{Methods}

The techniques for dissecting and staining flat teased preparations of the wall of the canal have already been described. They permit a detailed study of the structures comprising the trabecular wall, viewed from within the lumen of the canal. Dilute Indian ink was introduced beneath the coverslip of some of the wet preparations and direct observations were made of the movement of small particles through the wall of the canal. Thin paraffin sections were used to supplement the information obtained from the flat preparations. Fixation of some monkey eyes was performed by injecting the anterior chamber with formalin in vivo, while the animals were anaesthetized with Nembutal. Other eyes were fixed by immersion in formalin several hours after removal. Human material consisted of the anterior segments of surgical specimens which had been removed because of suspected intra-ocular tumours, and these were fixed by immersion in formalin after enucleation. One human eye was fixed by injecting formalin immediately after removal.

* This investigation was supported by a National Health Grant.

+ Received for publication March 4, 1960.

33 


\section{(A) Observations in the Monkey}

(I) Anatomy of the Trabecular Wall of the Canal.-Injections of formalin into the anterior chamber reduced but did not completely eliminate the formation of vacuoles in the corneal endothelium (Fig. 1a,b). However, the small vacuoles which remained visible in flat preparations could not be identified in paraffin sections, in contrast to the spongy appearance of the endothelium in eyes which were fixed by immersion in formalin immediately after enucleation, or after a delay of several hours.

(a)

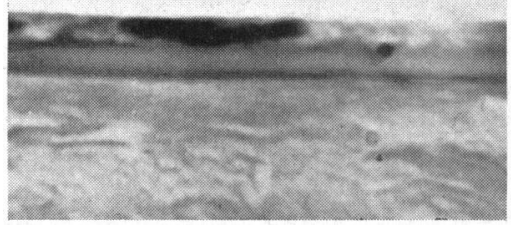

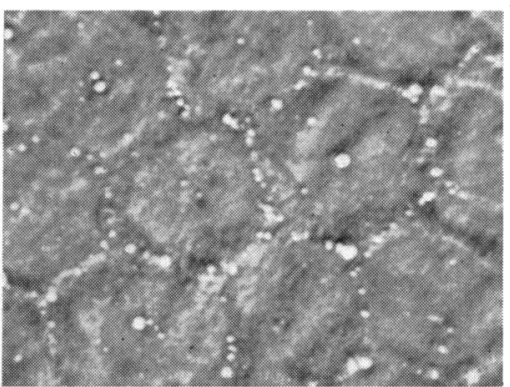

(b)

FIG. $1(a, b)$.-Paraffin section $(a)$ and flat preparation $(b)$ showing minimal vacuolation in corneal endothelium. (Monkey. (a) Mallory stain $\times 1,500 ;(b)$ Phase $\times 950$.)

The structure of the trabecular wall of Schlemm's canal was studied in several monkeys whose corneal endothelium showed only scattered fine vacuoles. In flat preparations the surface was grossly uneven with many grooves varying from 5 to $20 \mu$ in width and from 15 to $50 \mu$ in length. Many of the smaller grooves passed obliquely into the wall of the canal. The endothelium consisted in most areas of overlapping spindle cells which resembled the surface of a wicker basket. At the end of many of the nuclei were small cyst-like spaces 2 to $3 \mu$ in diameter. Careful focusing revealed a thin layer of cytoplasm separating many of the spaces from the lumen of the canal. Internally they appeared to communicate with spaces within the meshwork

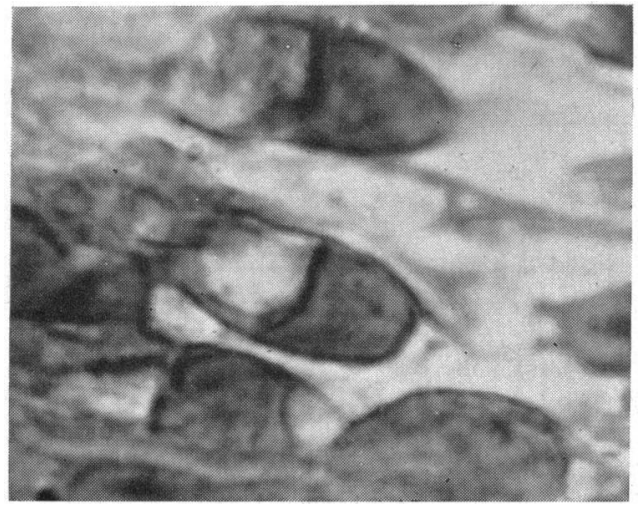
and many pigment granules were adherent to their walls. In other areas, where the endothelial cells were inclined obliquely or even perpendicularly to the wall of the canal, the spaces or defects were much larger (Fig. 2). Careful

FiG. 2.-Flat preparation showing narrow grooves and defects in the endothelial lining of Schlemm's canal. (Monkey. Polychrome methylene blue stain $\times 1,500$.) 
focusing showed the spaces to be part of a channel system running obliquely into the meshwork where connections took place with adjacent intertrabecular spaces or channels. It was difficult to obtain thin preparations from a monkey eye and clearly defined openings from the channels into the lumen of the canal could not be seen.

Additional information on the size and shape of the channels was obtained from a study of thin paraffin sections. The walls consisted of thin sheets of cytoplasm and the flattened nuclei frequently formed a third or more of the circumference of a channel. When cut at right angles they were circular with an internal diameter of 2 to $5 \mu$. When cut obliquely or parallel to their long axis many were found to be about $5 \mu$ in width and up to $10 \mu$ in length (Fig. 3, and Fig. 4, overleaf).

(a)

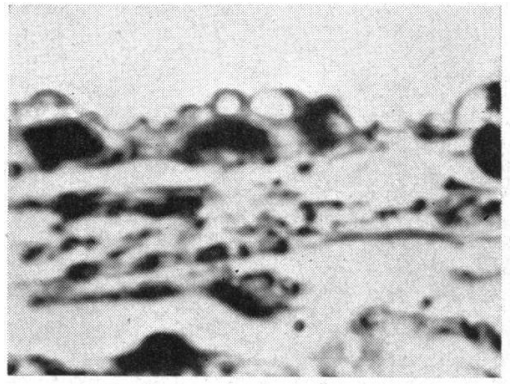

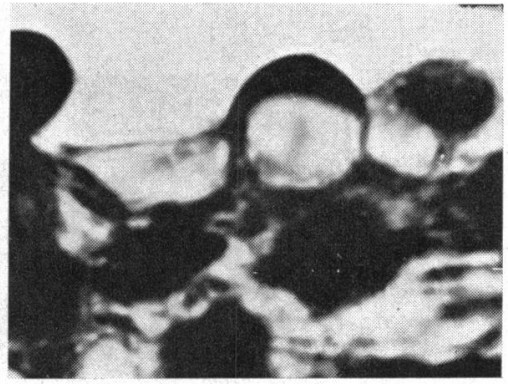

(b)

(c)

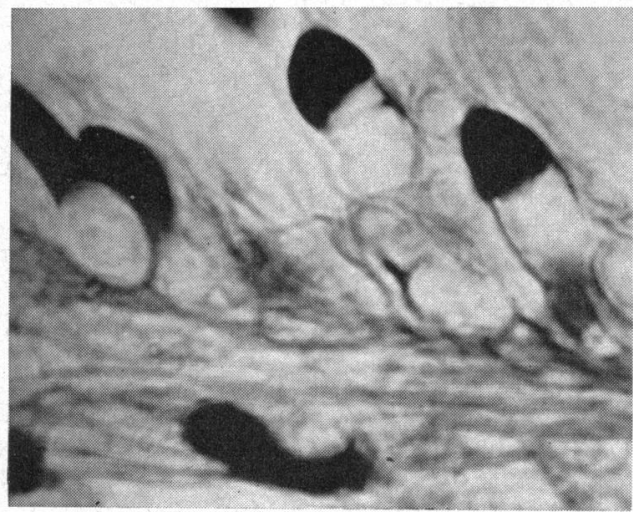

Fig. $3(a, b, c)$.-Paraffin sections showing channels in the endothelial lining of Schlemm's canal cut $(a, b)$ at right angles, and $(c)$ obliquely. (Monkey. Mallory stain $\times 1,500$.)

(2) Passage of Indian Ink through the Wall.-In order to establish where bulk flow took place through the wall of the canal, Indian ink was introduced under the coverslip of flat preparations and the Brownian movement of individual particles was studied by direct observation. Particles could be clearly seen disappearing into and emerging from the defects in the wall of the canal. When fluid was expressed from the tissue by racking down the microscope objective, particles frequently emerged from the meshwork through the defects in the wall of the canal, as the objective was raised. 
(3) Distribution of Red Blood Cells in the Wall.-A second piece of indirect evidence suggests that the channels are capable of conducting particulate matter. When the anterior chamber of an experimental animal is decompressed in vivo, a reflux of blood from the collector channels enters Schlemm's canal and many of the red cells find their way into the trabecular meshwork. They are a more physiological tracer than Indian ink and although their passage through the wall cannot be followed by direct observation their distribution can be studied in rapidly-fixed tissue. In flat teased preparations they were scattered in large numbers over the inner surface of the canal, and in meridional sections they were present on each side of the lining of the canal. However, the precise point at which they passed through the trabecular wall could only be determined from flat paraffin sections, where they were found crowding into the thin-walled channels seen previously in the flat teased preparations (Fig. 4).

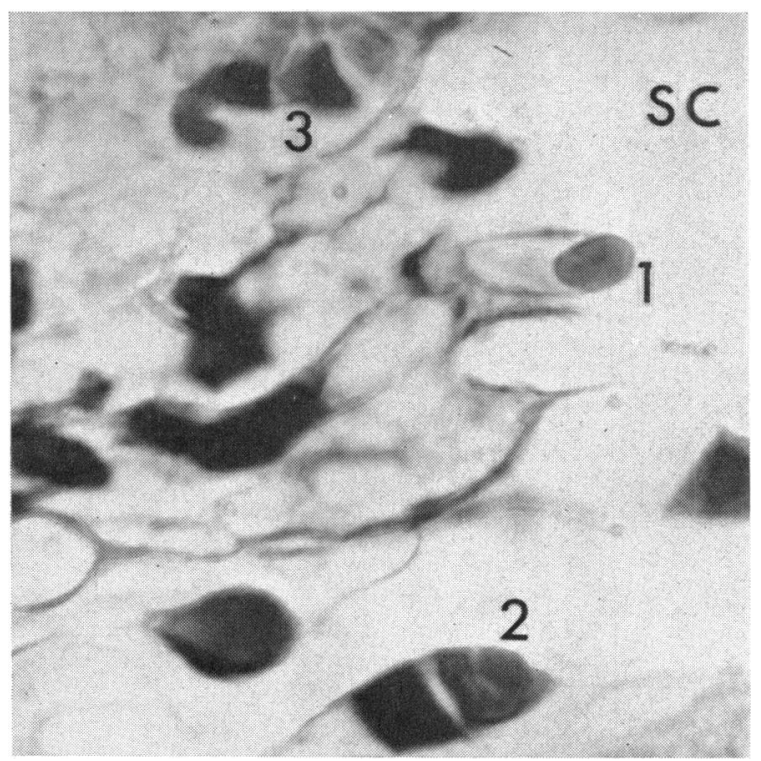

Fig. 4.-Paraffin section showing red blood cells: (1) entering a channel from Schlemm's canal (S.C.); (2) lying within a channel; (3) lying within the meshwork. (Monkey. Mallory stain $\times 1,500$.)

No significant difference could be found in the structure of the wall of the canal in a group of monkey eyes which were rapidly fixed and in a group in which fixation was delayed for several hours. Although ultra-microscopic changes may have been present, the grosser structure of the channels and defects appeared unchanged.

\section{(B) Observations in Man}

Flat preparations and paraffin sections of the trabecular wall of the canal in the human have been reassessed and compared with those in the monkey. The gross details of the large grooves and the spindle cell patterns displayed by the endothelium were very similar. The human meshwork is more brittle 
and at the edge of teased preparations small fragments were often found which were only two or three cells in thickness. This made it possible to define in greater detail the way in which adjacent channels communicated with each other and with the lumen of the canal.

(1) Size and Course of Channels.-These observations were made on an eye which had been fixed by injecting formalin at the time of enucleation, thus preventing the formation of vacuoles in the corneal endothelium. Numerous channels were found in the trabecular wall of the canal (Fig. 5), which followed a spiral course inclined at various angles to the wall of the canal (Fig. 6). It was possible to trace the outlines of the channels by fine adjustments of the microscope.

FIG. 5.-Flat preparation showing numerous channels in the endothelial lining of Schlemm's canal. (Human. Polychrome methylene blue stain $\times 750$.)
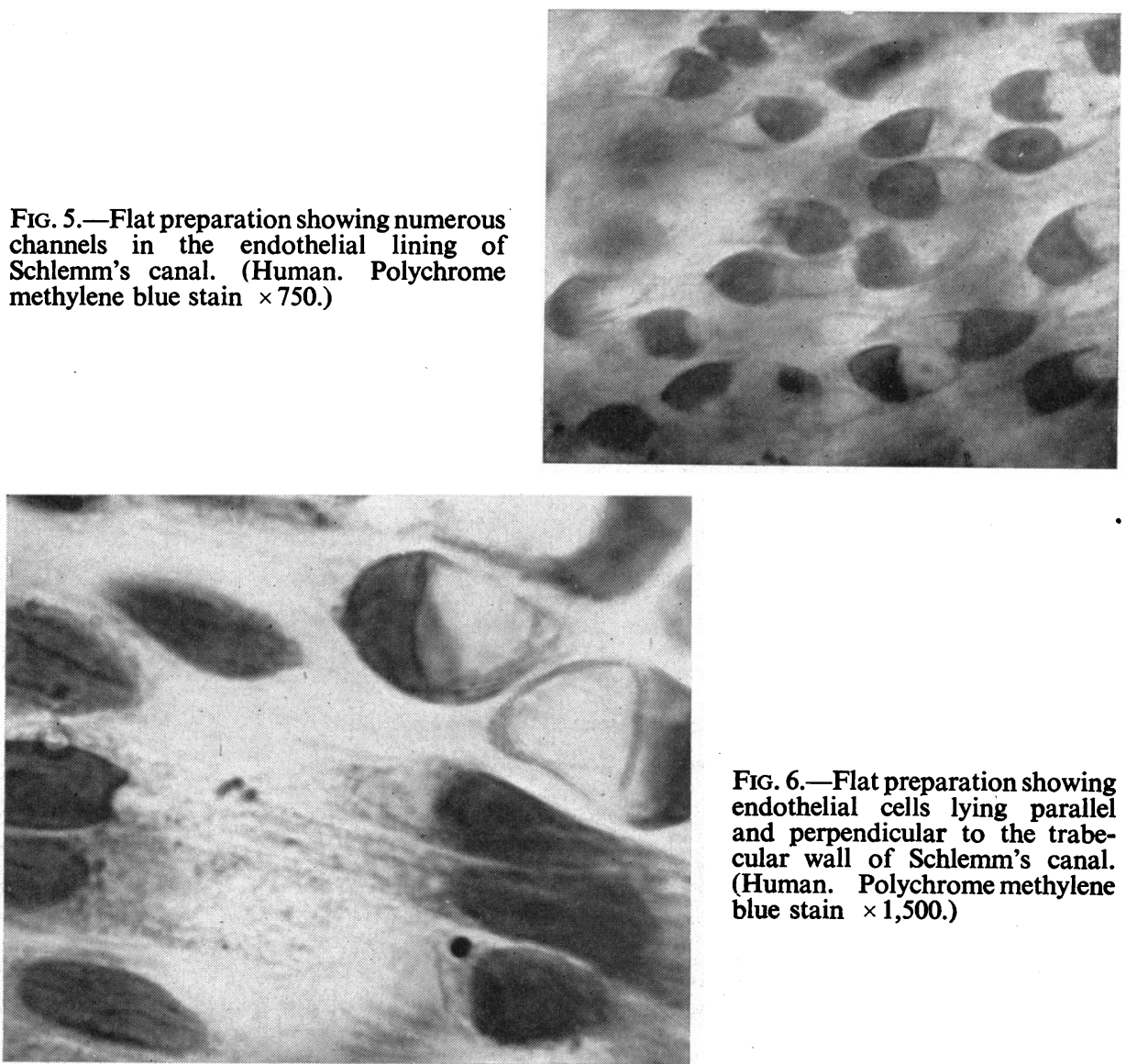

Fig. 6.-Flat preparation showing endothelial cells lying parallel and perpendicular to the trabecular wall of Schlemm's canal. (Human. Polychrome methylene blue stain $\times 1,500$.)

Their diameters varied from 2 to $6 \mu$, the majority being about $3 \mu$. Some channels, which were parallel to the wall, measured up to $15 \mu$ in length in one plane, and many others could be traced for distances of $10 \mu$. Communication between adjacent channels at slightly different levels took place by means 
of spiral openings (Fig. 7a,b). Although in photographs taken in a single plane they appear to be enclosed spaces within cells, the anatomy is more readily understood if they are regarded as spaces between cells possessing cytoplasm which is extremely attenuated.

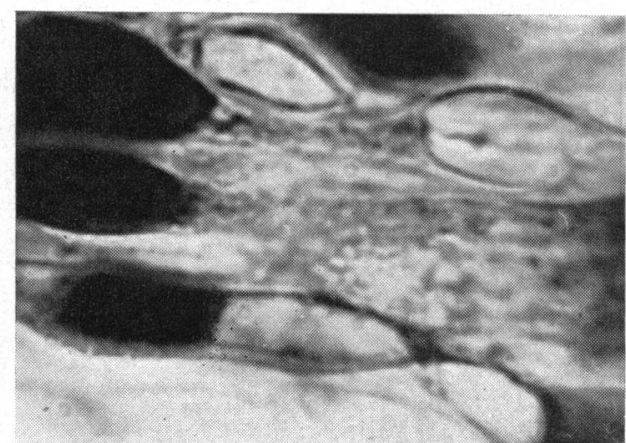

(a)

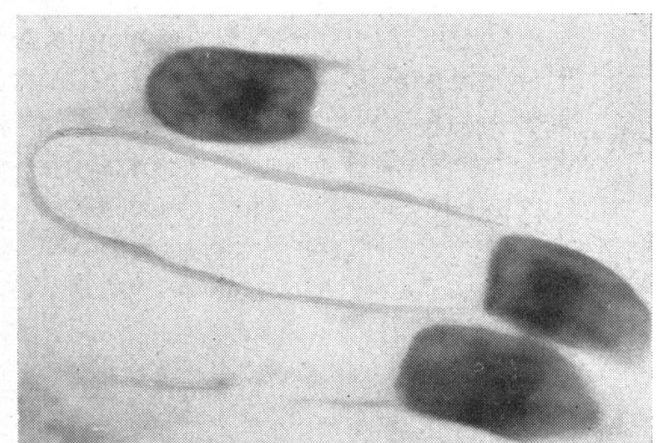

(b)

FIG. $7(a, b)$.-Flat preparation showing channels in the endothelial lining of Schlemm's canal. In $(a)$ a communication is present between adjacent channels. (Human. Polychrome methylene blue stain $\times 1,500$.)

(2) Communication between Channels and Schlemm's Canal.-The wall of the canal consists of numerous narrow grooves and ridges where the channels and cells lie parallel to the surface. Some of these channels communicate with the canal through spiral defects, and additional openings are probably overlooked because of the light microscope's limited resolving power. However, where the channels are steeply inclined to the floor of the canal, the walls stand out more clearly because of the greater depth of cytoplasm. It is then possible to see the openings and photograph the course of individual channels at different levels (Fig. 8).

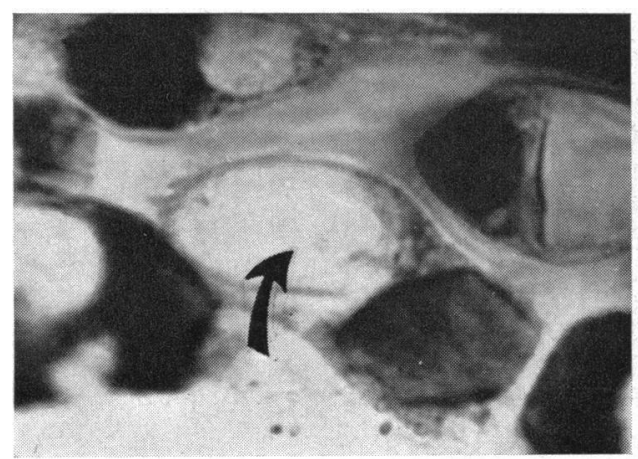

(a)

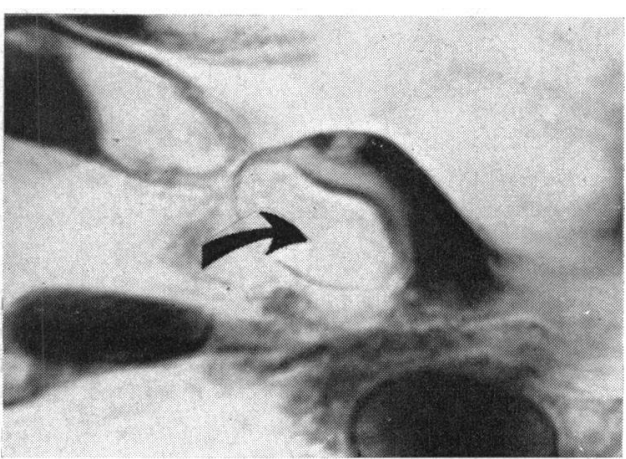

(b)

FIG. $8(a, b)$.-Flat preparation showing openings (arrows) into channels lying perpendicular to the wall of the canal. (Human. Polychrome methylene blue stain $\times 1,500$.) 
The openings have a spiral or ovoid configuration and measure up to $6 \mu$ in diameter. Some openings may lie in the sides of channels and because the angle of observation is limited they cannot be visualized. However, on several occasions as many as three openings have been seen in a single oil immersion field; consequently the total number must be a very high figure.

(3) Content of Channels.-The channels appear empty in the majority of specimens. However, where there has been a massive reflux of blood into the canal of Schlemm, one frequently finds serum clots adherent to the wall and filling the outermost channels. In one specimen several casts of the drainage channels resembling renal tubular casts were dislodged during dissection, but the material was not identified.

(4) Cell Boundaries around Channels.-In preparations stained with polychrome methylene blue the cell boundaries were invisible. However, in preparations stained with silver, the cell outlines were clearly seen (Fig. 9).*

FIG. 9.-Flat preparation showing deposition of silver at boundaries of endothelial cells. (Human. Silver stain $\times 750$.)

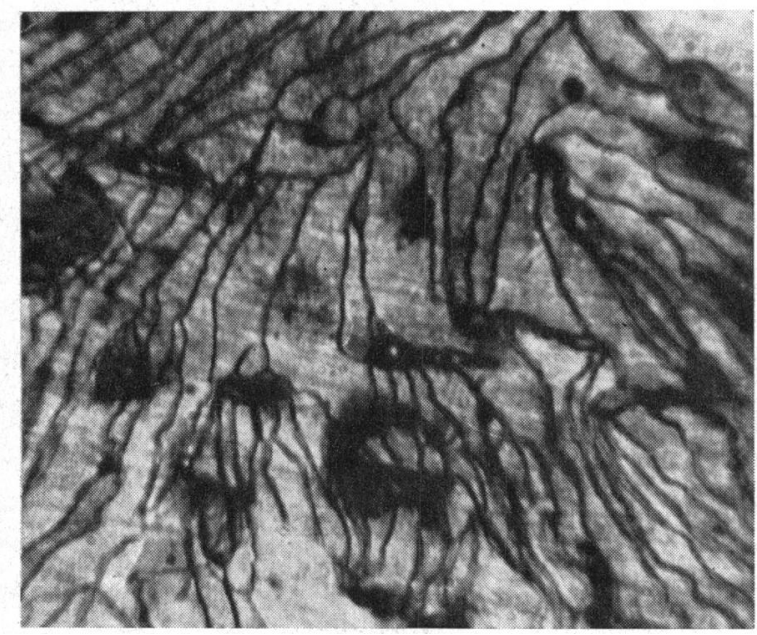

The pattern in places consisted of closely packed, nearly parallel lines, and probably corresponded to areas of the wall where the spindle cells resembled the surface of a wicker basket. Elsewhere the pattern looked more like the lead lines in a stained glass window and probably represented areas of the canal wall where the cells were inclined at a more acute angle to the surface.

(5) Reconstruction of Channels.-Plasticine reconstructions were made of certain channels and openings photographed at different levels. On the basis of these fragmentary reconstructions a composite model was prepared to illustrate the irregular inner wall of the canal, the oblique spiral openings,

* This microphotograph was provided by Dr. H. R. Hausler and Dr. T. Sibay. 
and the course taken by typical channels leading into the underlying meshwork (Figs 10 and 11, and Fig. 12, opposite). It would require a larger model to indicate the numerous possibilities.
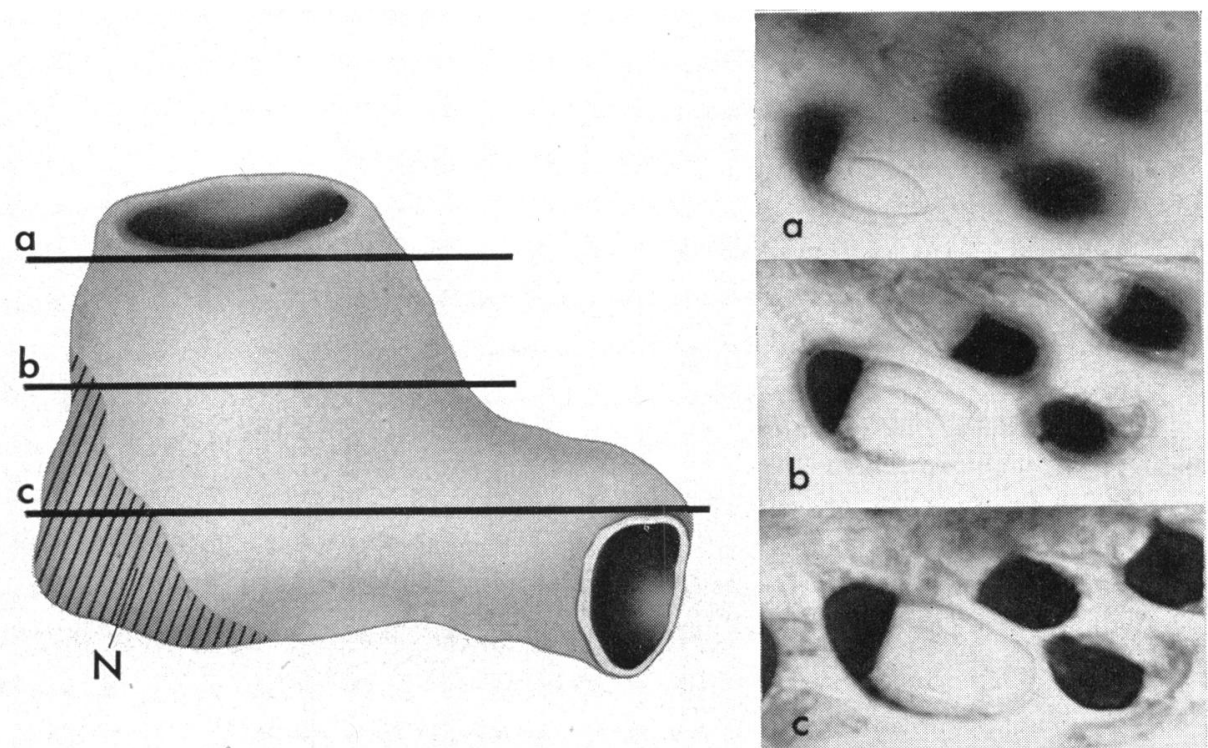

FIG. 10.-Reconstruction showing a single opening, channel, and nucleus $(\mathrm{N})$, based on microphotographs at different levels $(a-c)$ of a flat preparation. (Human. Reconstruction $\times 3,375$. Photomicrographs $\times 1,050$.)

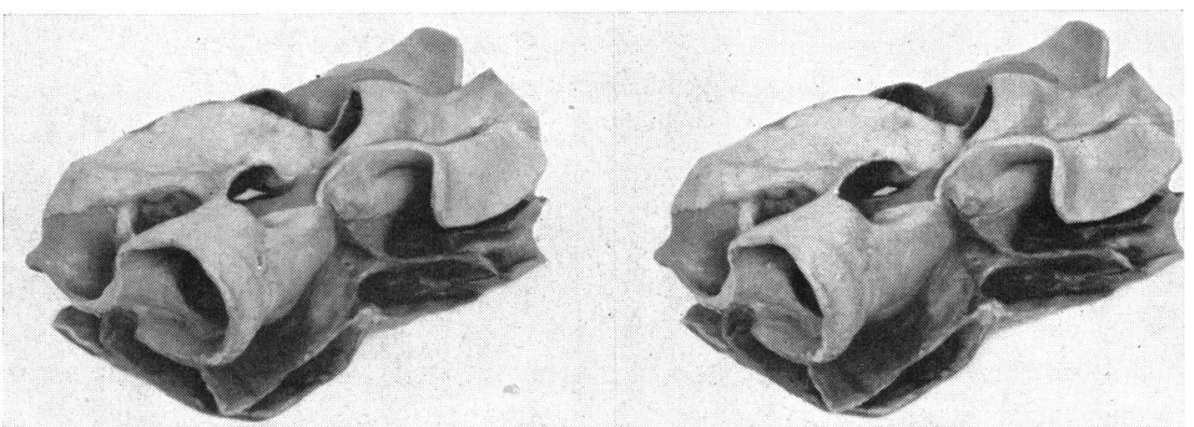

FIG. 11.-Stereo-pair of a composite reconstruction to demonstrate the openings and channels in the floor of Schlemm's canal in three dimensions.

\section{Discussion}

Sondermann (1930) believed that aqueous fluid entered the canal of Schlemm through large defects which he found in the trabecular wall in meridional paraffin sections. His observations have since been confirmed on many occasions, but it is unlikely that the defects he described represent the ultimate drainage openings into the canal, in view of the plexiform nature of 


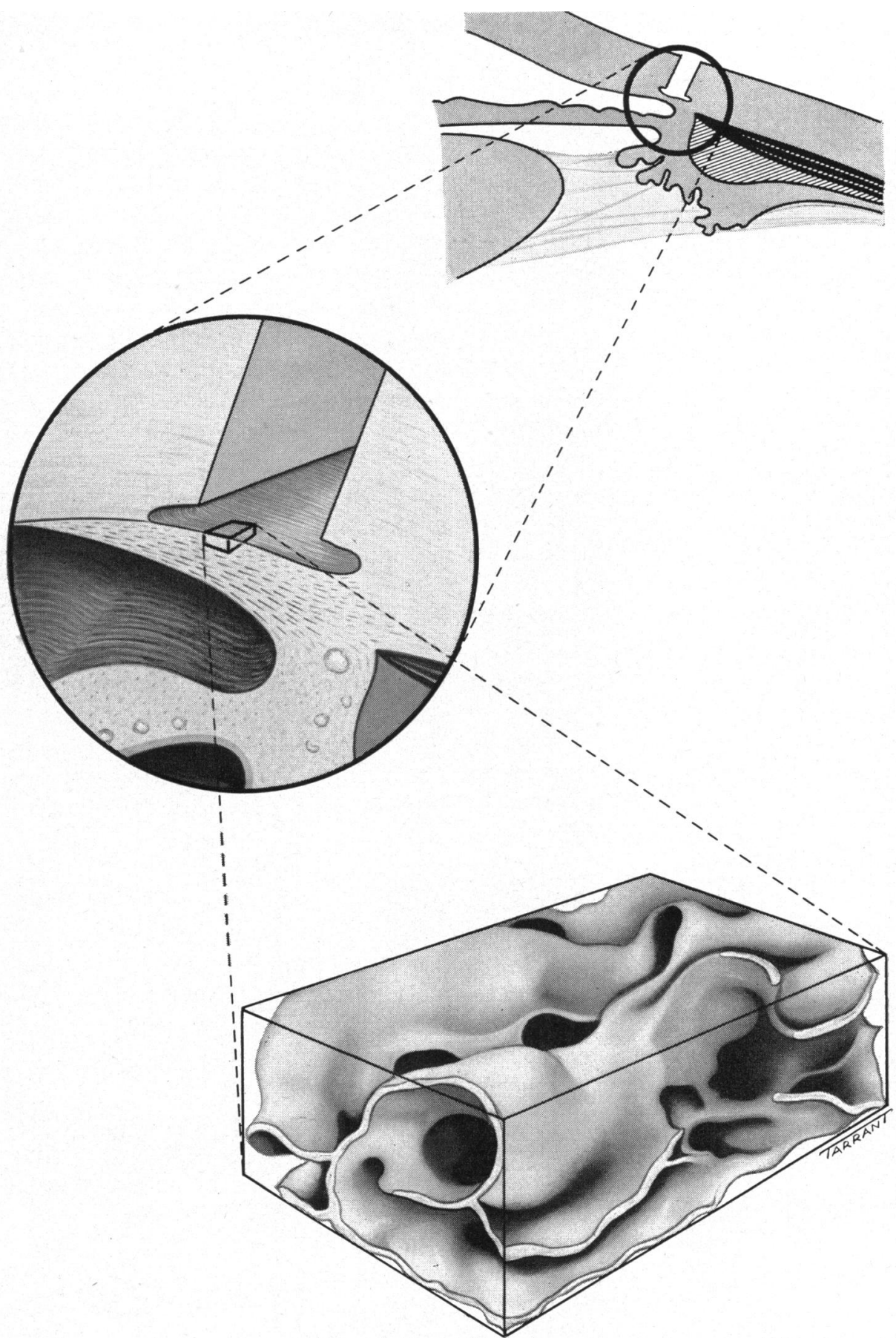

Fig. 12.-Diagrams showing the relationships of the spiral openings and channels between the endothelial cells which form the floor of Schlemm's canal. 
the meshwork in this region. The many deep grooves, which are seen on the surface of the trabecular wall in flat teased preparations, would present the appearance of openings when cut in cross-section. Unger and Rohen (1959) have recently illustrated these grooves in thick flat paraffin sections of the wall, and have shown that some of them are buried beneath the wall of the canal forming a true channel within the meshwork. These undoubtedly communicate with the canal through the larger openings, $10 \mu$ in diameter, identified previously in flat preparations of the wall (Speakman, 1959a).

The finding of gross vacuolation in the human corneal endothelium led to the initial conclusion (Speakman, 1959b) that similar post-mortem degeneration had also occurred in the trabecular endothelium, although Garron, Feeney, Hogan, and McEwen (1958), who first drew attention to the peculiar appearance of the endothelial cells lining the canal, did not believe postmortem change was responsible. The present study has shown that, whereas rapid fixation will prevent the development of gross vacuolation in the corneal endothelium, the defects in the endothelium lining the canal wall remain, and do not change in appearance even when fixation is delayed for several hours. Consequently the previous suggestion that they represent a post-mortem artefact must be modified.

Unger and Rohen (1959) thought that the trabecular wall of Schlemm's canal and the large grooves or channels in this region were lined by an intact layer of endothelial cells, except at the beginning of their afferent venous channels, which were compared to the venous roots in the spleen of cats and pigs. Garron and others (1958) and Garron and Feeney (1959) were also unable to demonstrate direct openings through the wall of Schlemm's canal, although Garron and Feeney (1959) illustrated red blood cells lying in the defects or "vacuoles" in the endothelial lining, and described red cells incarcerated in the wall. In view of the very marked irregularity of the surface, it is difficult in single thin sections to differentiate a narrow groove between cells from a true opening. Holmberg (1959), using thin serial sections, has obtained more convincing evidence that direct pathways, 0.5 to $1.5 \mu$ in diameter, exist between the trabecular spaces and Schlemm's canal through the "vacuoles" in the endothelial cells. However, the actual size distribution of the openings and channels and their variable spiralling course cannot be determined from such small fragments of tissue. Flat teased preparations preserve the third dimensional relationships and show that the endothelial lining is perforated by many openings up to $6 \mu$ in diameter. The latter mark the exit point of numerous small channels between the endothelial cells which open into the floor of Schlemm's canal and communicate with spaces in the underlying trabecular meshwork.

The direct observation of Indian ink particles travelling through the channels demonstrates that they are channels and not enclosed spaces. The finding of uveal pigment granules adhering to the walls, and the identification of red blood cells trapped inside the channels at various levels, offer good 
evidence that bulk flow may take place in either direction through these structures in the living eye.

In the past the trabecular meshwork has been divided into an inner uveal portion, consisting of a lacelike network of fibres which stretch from Schwalbe's line to the root of the iris, and an outer corneo-scleral portion, composed principally of broad collagen bands which encircle the eye between the corneal stromal fibres and scleral spur. Recent studies with the light and electron microscope have disclosed a third zone, approximately $10 \mu$ in depth, on the outer aspect of the trabecular meshwork forming the floor of Schlemm's canal, which is composed almost exclusively of endothelial cells. It would seem appropriate to name this area the endothelial meshwork in order to draw attention to this important part of the outflow pathway and to emphasize its principal structural component.

\section{Conclusion}

Numerous openings up to $6 \mu$ in diameter have been found in the endothelial lining of Schlemm's canal in the human eye. The openings are the exit points of spiralling channels 5 to $10 \mu$ in length, which communicate with the underlying trabecular meshwork and represent the final pathway taken by the aqueous fluid in reaching the lumen of the canal. It is suggested that this area be designated the endothelial meshwork.

I am grateful to Prof. Norman Ashton for valuable advice during the preparation of this paper. I am also indebted to Miss E. FitzGerald for secretarial assistance, and to the Medical Illustration Department of the Institute of Ophthalmology for providing the stereo-photographs and drawings.

\section{REFERENCES}

Garron, L. K., and Feeney, M. L. (1959). Arch. Ophthal. (Chicago), 62, 966.

G. 27. Holmberg, A. (1959). Arch. Ophthal. (Chicago), 62, 956.

SondermanN, R. (1930). v. Graefes Arch. Ophthal., 124, 521.

SPEAKMan, J. S. (1959a). Brit. J. Ophthal., 43, 129.

(1959b). Ibid., 43, 139.

Unger, H. H., and ROHEN, J. (1959). Amer. J. Ophthal. 48, 204. 